\title{
Direct Transference of Emergence Profile in Implant Supported Fixed Bridges
}

\author{
Real Voltas F, Scalcione R, Figueras-Alvarez 0* and Romano Cardoso E \\ ${ }^{1}$ Department of Prosthodontics, School of Dentistry, Universitat Internacional de Catalunya Spain
}

Submission: : November 25, 2016; Published: December 13, 2016

*Corresponding author: Oscar Figueras-Alvarez, Department of Prosthodontics, School of Dentistry, Universitat Internacional de Catalunya, Barcelona, Spain, Tel: +34935042000; Email: ofigueras@uic.es

Abstract

An implant restoration in the anterior area requires careful management of soft and hard tissues due to biological, functional and aesthetics requisites. The use of provisional restorations is necessary to create a proper emergence profile and mimic the natural dentition and their supporting gingival tissues. It also contributes to the health of peri-implant tissues and facilitates to perform a correct oral hygiene. Transferring the shape of the created emergence profile is key to fabricating the proper final restoration. This report describes a direct technique to take a final impression of an implant-supported bridge. Impression abutments and flowable composite resin were used to fill the gingival emergence profile and obtain a precise master model cast.

Keywords: Emergence profile; Implant; Pontic; flowable composite resins

\section{Introduction}

The esthetic success of implant supported restorations is given by the form and health of the surrounding peri-implant and pontic site tissues. To obtain predicted outcomes, definitive restorations must follow the optimal soft tissue modification established intraorally by provisional restorations [1]. After the removal of the implant healing caps the geometry of gingiva is circular. Specifically, incisors have a triangular gingival profile [2]. This shape can be modeled by the weekly addition or removal of composite resin to the provisional restoration over a period of 1 or 2 months, simulating the shape of the final restoration. A screw retained provisional restoration is the indicated because of the cement absence. However, an unfavorable screw access could be an indication of cement retained provisional [3]. Once a natural emergence profile that pleases both the clinician and the patient is achieved (Figure 1), a final impression can be taken to obtain the master cast [4].

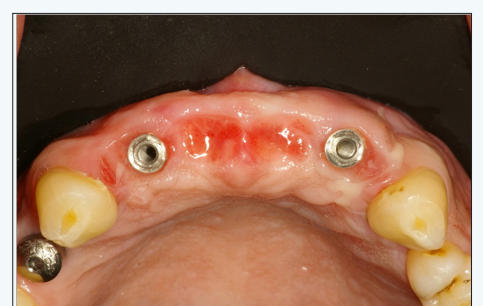

Figure 1: Emergence profile achieved by the use of provisional restorations.
The soft tissue contour created during the provisional restoration must be preserved on the definitive model to have an accurate communication with the laboratory [1]. Direct and indirect techniques are used to transfer the emergence profile. Indirect techniques need the fabrication of custom impression copings, having the inconvenient of cost and chair time. Direct techniques use the interim restoration as an impression coping or an in situ registration of the surrounding tissues. The main objective of this article is to describe a technique that preserves and reproduces the emergence profile of both implants and pontics, by intraoral application of a flowable photo-polymerized composite during the final impression procedure.

\section{Technique}

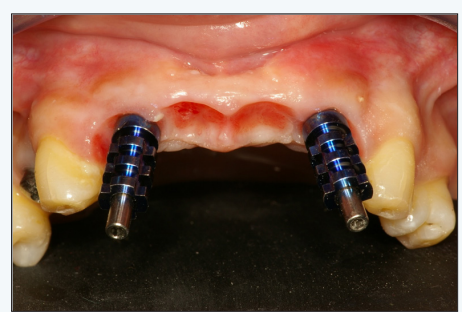

Figure 2: Impression transfers are placed in the implant connections.

a) After removing the provisional restoration, a standard impression abutment is placed in the implant connection (Figure 2). An intraoral radiograph may be necessary to 
assure the correct fit of the impression abutment on the implant.

b) The space between the impression post and the gingiva is immediately filled with flowable composite resin in layers of $2 \mathrm{~mm}$ maximum thickness (Filtek Supreme XTE Flowable Restorative; $3 \mathrm{M}$ ESPE). The resin was subsequently polymerized (Figure 3).

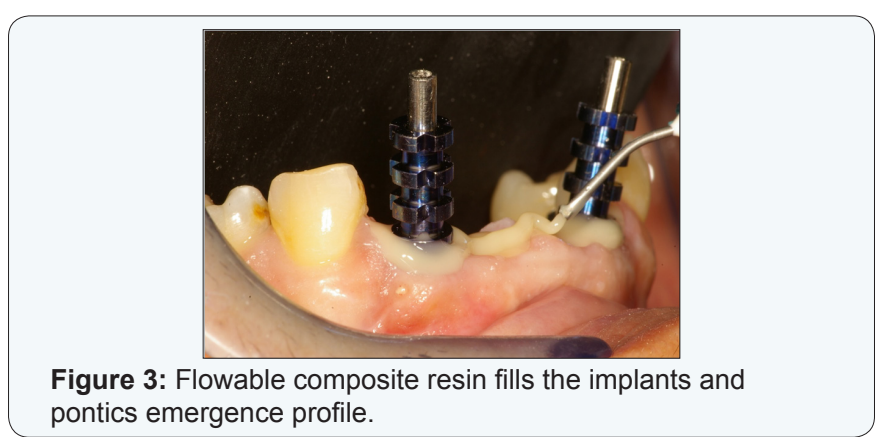

c) The same procedure is utilized in the ovoid pontic of the gingiva to fill and copy the emergence profile. The flowable composite resin in the pontics area must join with the composite resin around the impression abutments. Thus, the entire composite will come out as a block when taking the final impression (Figure 3).

d) Once all of the flowable composite resin has been placed and polymerized, a final impression with polyvinylsiloxanes or polyethers was taken to obtain a final model cast (Figure 4).

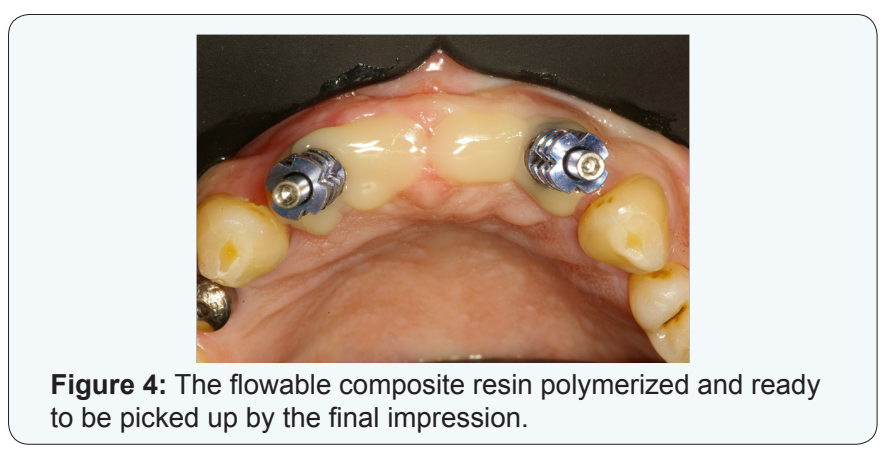

\section{Discussion}

Several published techniques have been used for customized impression abutments [1,4,5]. Extraoral and intraoral procedures can be used to customize abutments during unitary restorations, but to our knowledge the literature only describes extraoral procedures in restorations involving implants and pontics [5]. These extraoral procedures may require extended chair time. Moreover, soft tissues may collapse intraorally while the abutments are being customized extraorally. This may cause discomfort to the patient when the customized impression abutments are placed on the implants. However, the use of customized healing abutments can avoid tissue collapse [6]. The direct application of flowable composite resin to the gingival [4] is advantageous, in that the final impression is taken directly in the patient's mouth. This technique allows the reproduction of the shape of the pontic intaglio surface form and the implant prosthesis, reducing procedure time and minimizing gingival collapse. This is a fast and accurate method of transferring the correct shape of the created emergence profile to fabricating the final restoration.

\section{References}

1. Ntounis A, Petropoulou A (2010) A technique for managing and accurate registration of periimplant soft tissues. J Prosthet Dent 104(4): 276-279.

2. Wittneben JG, Buser D, Belser UC, Bragger U (2013) Peri-implant soft tissue conditioning with provisional restorations in the esthetic zone: the dynamic compression technique. Int J Periodontics Restorative Dent 33(4): 447-455.

3. Hegyi KE (2016) Direct method of registering periimplant soft tissue forms for implant-supported fixed dental prostheses. J Prosthet Dent 115(3): 267-270.

4. Papadopoulos I, Pozidi G, Goussias H, Kourtis S (2014) Transferring the emergence profile from the provisional to the final restoration. J Esthet Restor Dent 26(3): 154-161.

5. Spyropoulou PE, Razzoog M, Sierraalta M (2009) Restoring implants in the esthetic zone after sculpting and capturing the periimplant tissues in rest position: a clinical report. J Prosthet Dent 102(6): 345-347.

6. Figueras-Alvarez O, Real-Voltas F (2015) A method of temporarily preserving the emergence profile in implant dentistry. J Prosthet Dent 113(5): 491-492.

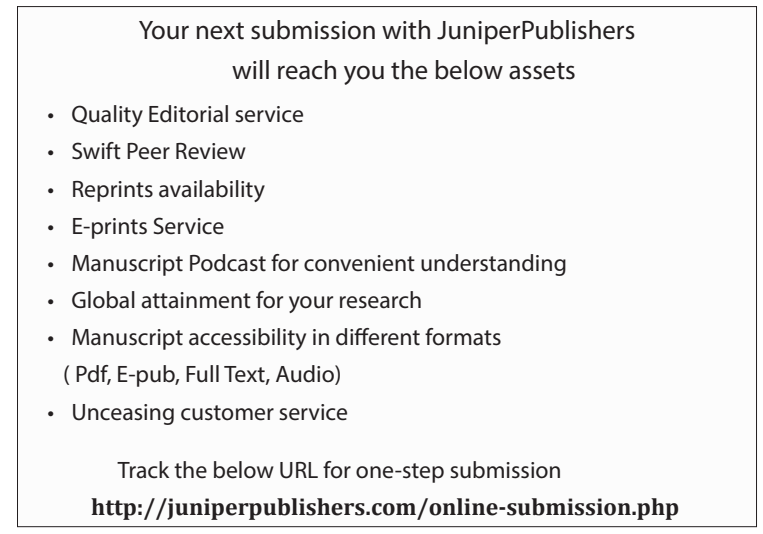

